\title{
GESTIÓN DE DOCUMENTOS: SONRISAS Y LÁGRIMAS
}

\section{Adela d'Alòs-Moner}

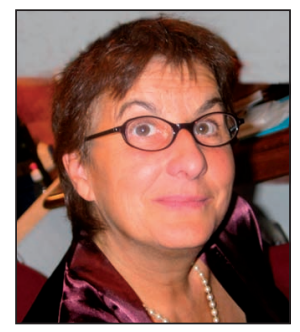

Adela d'Alòs-Moner, socia fundadora de Doc6, tiene una larga trayectoria tanto en centros públicos como privados. Ha sido directora de la biblioteca de ciencias de la Universitat Autònoma de Barcelona y coordinadora de distintos proyectos europeos. Ha impartido clases en varias universidades y ha sido presidenta del Col-legi Oficial de Bibliotecaris i Documentalistes de Catalunya (Cobdc). Miembro del Grupo ThinkEPI.

Doc6, Consultores en Recursos de Información Barcelona y Madrid aalos@doc6.es http://www.doc6.es

\section{Resumen}

Basados en la experiencia en proyectos de gestión de documentos y del conocimiento, se presentan los diez aspectos más importantes que hay que tener en cuenta para llevar a cabo con éxito un proyecto de mejora de gestión de documentos en una organización.

\section{Palabras clave}

Gestión de documentos, Gestión del conocimiento, Organizaciones, Empresas, Pautas, Recomendaciones.

\section{Title: Document management: the ups and downs}

\section{Abstract}

Based on experiencies in document and knowledge management, the article explains ten aspects that must be considered in order to carry out successfully a project on document management.

\section{Keywords}

Document management, Knowledge management, Organisations, Companies, Guidelines, Recommendations.

D’Alòs-Moner, Adela. "Gestión de documentos: sonrisas y lágrimas". El profesional de la información, 2011, julio-agosto, v. 20 , n. 4 , pp. $456-459$.

\section{Introducción}

En 2002 escribí el artículo Intranets: sonrisas y lágrimas ${ }^{1}$. En aquel momento las intranets se estaban expandiendo en las organizaciones y empezaba a haber un "corpus" de experiencias que permitía hacer una reflexión sobre cómo debía implementarse una intranet para garantizar su éxito.

Han pasado casi diez años y hoy un tema "estrella" es el de la gestión de la documentación en las organizaciones. También en este caso tenemos suficientes experiencias acumuladas para poder realizar unas primeras reflexiones o recomendaciones que faciliten que un proyecto de gestión de documentos pueda obtener los objetivos esperados. Me permito pues la licencia de usar la misma expresión para este articulo "Gestión documental: sonrisas y lágrimas".

¿Qué preocupa a las organizaciones? ¿Qué sucede hoy en muchas de ellas, en lo que concierne a la información y la documentación? Les preocupa la pérdida o la dificultad de acceso a documentación crítica, indispensable, por ejemplo, para dar respuesta a una auditoría. También les preocupan las "pérdidas de tiempo" o las horas que dedica el personal a buscar información o a reelaborar documentos que ya existen. Preocupa que cuando un empleado deja la organización es prácticamente imposible encontrar los documentos en su ordenador, o que si no está por cualquier motivo, sea complicado encontrar un anexo importante que se ha recibido por correo electrónico.

Al mismo tiempo se empieza a constatar una preocupación creciente por la "legibilidad" y el acceso futuro a la documentación. Esta inquietud es resultado, muy a menudo, de alguna penosa experiencia por la dificultad de recuperar un documento redactado hace apenas 8 ó 10 años o de visualizar un vídeo del que ya no se dispone del equipo de reproducción.

La necesidad de gestionar la documentación es un hecho evidente y responde fundamentalmente a dos motivos: 
por una parte, al crecimiento exponencial de información -se dice que un 60\% cada año- en múltiples formatos y, por otra, a la multiplicidad y generalización en el uso de los dispositivos de recepción y de generación de documentos, resultado de la expansión e impacto de las tecnologías -internet, ordenadores personales, PDAs...-.

Sobre el crecimiento de la información se ha escrito mucho últimamente. El informe Data, data everywhere de Kenneth Cukier publicado por The economist en febrero de $2010^{2}$ resume en pocas páginas la situación actual y las perspectivas en el próximo futuro. Usa la expresión "monstruosa cantidad de datos" y cita a Joe Hellerstein, de la Universidad de California, que habla de "la revolución industrial de los datos". Constata también, como a menudo se comenta en otros artículos, que la información que se genera es muy superior a la capacidad de almacenamiento disponible.

Esta realidad global tiene su expresión en las empresas que se encuentran cada vez más "perdidas" en la inmensidad de datos y documentos que se acumulan en sus redes locales y ordenadores personales. Necesitan localizar con agilidad y fiabilidad documentos que generan y reciben pero, sobre todo, necesitan dar sentido a la gran cantidad de datos e información que se encuentra en ellos.

Poder acceder a los documentos en el momento en que se necesitan y preservar aquellos a los que deberá ser posible acceder en un futuro, son elementos esenciales en el uso y capitalización de la información.

Para asegurar la eficiencia de las organizaciones y su capacidad innovadora y la plena integración de la información y la documentación en sus procesos de actividad, es necesario que los documentos y sus contenidos estén:

- Accesibles, donde, cuando y por quien sea necesario.

- Usables, de manera que además de accesibles sean interpretables para las personas que deben hacer uso de ellos.

- Integrados, en función de las necesidades y requerimientos de la empresa y sus trabajadores.

- Actualizados, según el desarrollo de la organización y su producción documental, para garantizar su permanente vigencia.

¿Cuáles son los errores que se producen con más frecuencia? ¿Qué aspectos debemos contemplar en un proyecto de gestión de documentos? Podríamos concretar estos diez:

\section{Decálogo de los proyectos de gestión documental}

\section{Implicación de la dirección}

Es evidente que a la mayoría de los responsables de las organizaciones les preocupa la situación de la documentación pero también es cierto que, en muchos casos, consideran -erróneamente- que éste es un tema "informático"; es decir, consideran que la solución pasa por implementar un sistema automatizado.

Que los directivos entiendan que la solución no es informática -o no es únicamente informática- no siempre es fácil pero es imprescindible. En la mayoría de casos hace falta un

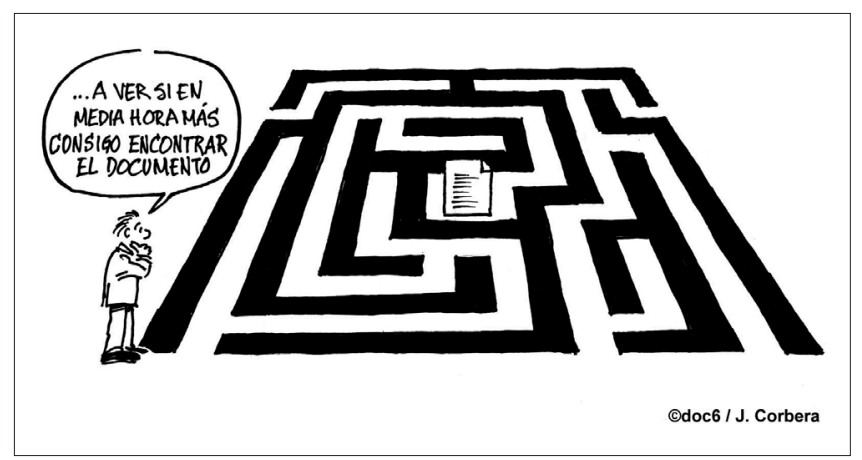

trabajo de explicación, de guía y asesoramiento que les permita visualizar los beneficios que un proyecto bien diseñado de gestión de la documentación puede aportar.

El primer aspecto es, pues, la implicación de la dirección, algo indispensable en un proyecto de estas características ya que comporta siempre cambios en la cultura de la empresa, en los procedimientos y muy a menudo en la propia estructura organizativa.

Intentar llevar a cabo un proyecto de mejora de la gestión de la documentación sin una implicación clara y directa de la dirección quizá permita pequeños avances, pero pocas veces representará una mejora sensible.

\section{Entender la organización y tener una visión amplia del entorno en la que ésta opera}

La documentación es un claro reflejo de las actividades y, por tanto, para llevar a cabo cualquier proyecto de mejora de gestión de la documentación es forzoso entenderlas. Comporta conocer la misión de la entidad, sus objetivos, sus prioridades, sus procesos clave de negocio, etc.

Un proyecto de mejora de gestión de la documentación debe abordar en profundidad los flujos de trabajo y los procedimientos y tener en cuenta los roles de las distintas personas implicadas en los mismos.

Pero además de "la propia entidad" se hace necesario un conocimiento del entorno, de datos macroeconómicos y microeconómicos, del mercado, de tendencias, del entorno legal y normativo en el que opera, de la competencia...

Esta visión más amplia es obligada si se quiere ir más allá de "ordenar" los documentos; la aportación de valor reside en la habilidad de "dar sentido" -por ejemplo, con el uso de taxonomías que integren bajo un único concepto documentos asociados a procesos de actividad distintos; $y$, sobre todo, en la capacidad de contribuir al valor de uso a la documentación, de convertir la información en conocimiento que facilite la toma de decisiones.

\section{Sumar visiones y conocimientos. Trabajo en equipo}

El trabajo aislado no tiene cabida en un proyecto de gestión de la documentación. Depende de cada organización el decidir quién deberá formar parte de un equipo de trabajo.

Los responsables del departamento de gestión de documentos, información o archivo, de calidad, tecnología, organización, recursos humanos o gerencia son los más habituales, y constituyen equipos multidisciplinares con los que es posible obtener mejores resultados en menos tiempo. 


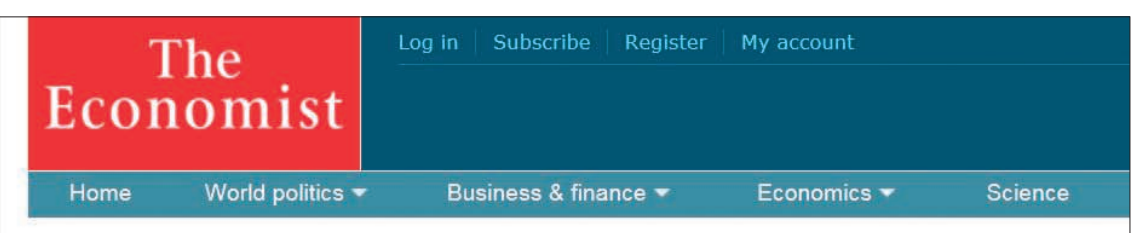

A special report on managing information

\section{Data, data everywhere}

Information has gone from scarce to superabundant. That brings huge new benefits, says Kenneth Cukier (interviewed here)-but also big headaches

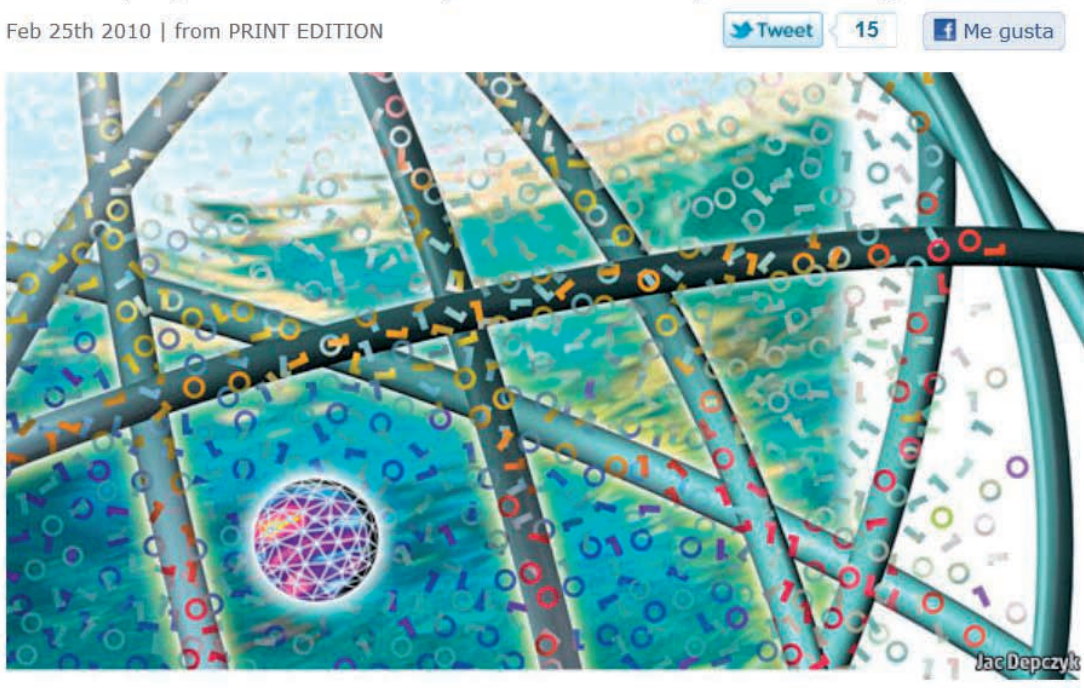

WHEN the Sloan Digital Sky Survey started work in 2000, its telescope in New Mexico collected more data in its first few weeks than had been amassed in the entire history of astronomy. Now, a decade later, its archive contains a whopping 140 terabytes of information. A successor, the Larae Svnoptic Survev Telescode. due to come on stream in http://www.economist.com/node/15557443

Para avanzar es indispensable la implicación de distintos perfiles: el "solo ante el peligro" se convierte en algo realmente peligroso y, sobre todo, poco eficiente.

\section{Asignación de responsabilidades}

El hecho de llevar a cabo un trabajo en equipo no comporta que no deba haber asignación clara de responsabilidades. Según la dimensión de la organización estas deberán situarse a distintos ámbitos: un responsable global de gestión documental y además, por ejemplo, responsables departamentales. Un aspecto fundamental es que cada uno de los trabajadores sea consciente de su responsabilidad con los documentos que genera o recibe.

Un proyecto en el que haya actividades o tareas indefinidas y sin asignación de responsabilidades concretas necesita su reconsideración global.

\section{Formación}

Un proyecto de gestión de la documentación comporta necesariamente dedicar horas a formar al personal en los nuevos procedimientos y pautas de trabajo $\mathrm{y}$, eventualmente, en las herramientas tecnológicas que se puedan implementar. Es esencial que las personas en la organización entiendan y se sientan cómodas con los nuevos procedimientos de trabajo. La formación contribuye en gran medida a superar "resistencias al cambio" que casi siempre se producen. nica. blica.
Además de la formación debe asegurarse el soporte y apoyo al personal para solventar dudas o dificultades con las que normalmente se enfrentan las primeras semanas.

Un error que se comete a menudo es, precisamente, el de no haber contemplado el tiempo necesario para formar al personal ni el soporte y apoyo que hay que seguir dando de manera continuada, hasta que los nuevos procedimientos están totalmente interiorizados.

\section{Cultura corporativa. Comunica- ción}

Los aspectos relacionados con la cultura corporativa son decisivos. Para que un sistema de gestión documental sea realmente efectivo debe haber una cultura orientada a compartir. Crear y establecer pautas para romper "silos" y compartimentos estancos. La comunicación tiene aquí un papel relevante ya que debe servir para transmitir una visión global de los beneficios a obtener que vaya más allá del propio ámbito de actuación.

Definir y concretar una política corporativa y que el personal en su globalidad la asuma, no es un tema fácil.

Los cambios en las organizaciones los realizan las personas. Sin ellas, sin su complicidad, sin su implicación, un proyecto de gestión documental posiblemente acabará atascándose. Un aspecto que a menudo no se tiene suficientemente en cuenta es la diversidad de realidades de las personas de la organización en cuanto a sus habilidades y actitudes. Lo que puede haber funcionado en una organización no tiene porque también operar positivamente en otra.

\section{Herramientas de gestión}

En un sistema de gestión de documentos se utilizan instrumentos como son los cuadros de clasificación, los calendarios de conservación y eliminación, vocabularios de metadatos, etc. Usados tradicionalmente para gestionar los documentos en soporte papel, se han ido modificando para adaptarse a la nueva realidad de la documentación electró-

Aunque parece evidente que antes de la selección e implementación de cualquier software deberían haberse contemplado todos las facetas conceptuales: desde cuadros de clasificación y mapas documentales a aspectos relacionados con los conocimientos de las personas, es frecuente que se decidan previamente programas software que después son difícilmente adaptables a la realidad de la organización. Por ejemplo, no se prevén aspectos relacionados con la preservación y migración de documentos, o con su adecuación a la normativa, como la Ley 11/2007 en la administración pú- 


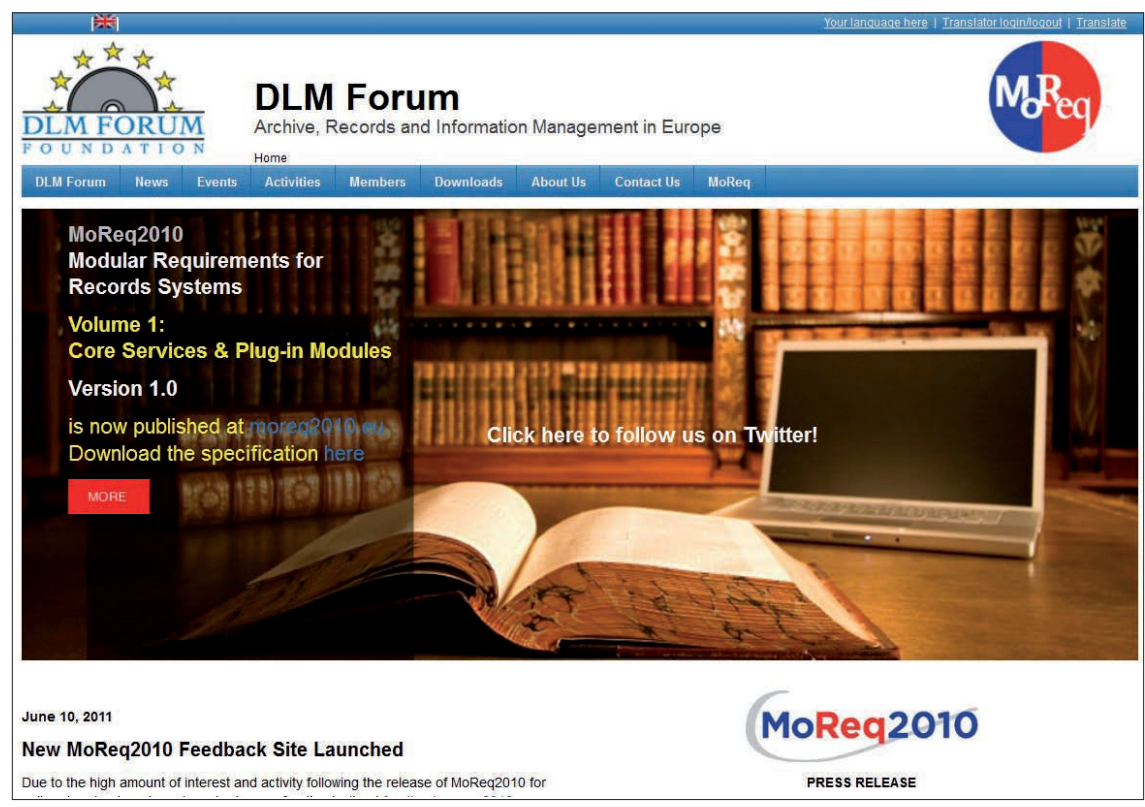

http://www.dlmforum.eu

Seguramente el error más frecuente es implementar un software de gestión documental sin haber realizado una toma de requerimientos funcionales que tengan en cuenta el uso de un cuadro de clasificación, una taxonomía, los modelos de metadatos a aplicar, etc. MoReq2 (Model requirements for the management of electronic records $)^{1}$ es una referencia importante ya que establece los requisitos que debe cumplir cualquier programa de gestión de documentos.

Hay que evitar realizar una aplicación mimética de los esquemas "clásicos" estudiados antaño en archivos o en documentación. Posiblemente no encajen con la cultura organizativa o con lo que la dirección de la empresa y las personas esperan. Seguramente será necesario cuestionarse algunas de las bases teóricas aprendidas.

\section{Un proyecto global. Pequeños avances}

Llevar a cabo un proyecto de gestión de documentos en cualquier organización requiere tiempo. Es por ello que hace falta -una vez diseñado y enmarcado el proyecto global, sus beneficios, resultados, etc.-, ir avanzando por fases sobre todo en aquellos departamentos o áreas con las personas más proactivas, que pueden actuar como elemento de referencia para los demás.

Es importante realizar un planeamiento que permita pequeños avances iniciales, que producen una visión positiva en la organización, porque esto desencadena buenas condiciones y una predisposición para posteriores etapas y avances.

\section{Resolver los problemas de hoy, anticiparse a los de mañana}

Un proyecto de gestión de documentos debe, en primer lugar, dar respuesta a los problemas y disfunciones de la orga- nización hoy. Los resultados, los beneficios deben ser evidentes y deberían poderse cuantificar.

Si hay algo que demandan las organizaciones, especialmente en momentos de crisis y de dificultades, es la mejora de la eficiencia y, en este aspecto, una correcta gestión de la documentación, y por ello, del conocimiento corporativo, es un aspecto clave que puede aportar beneficios a corto plazo. Pero más allá es indispensable pensar en "mañana", identificando claramente los riesgos del sistema y su sostenibilidad. Especialmente en temas como la preservación hay que ser extremadamente cauto y riguroso para proponer iniciativas que puedan ser viables en el tiempo.

\section{Marco normativo}

Sitúo este aspecto en último lugar, aunque no por ello es menos importante. Además del marco normativo y legal en el que actúa la organización, para diseñar un sistema de gestión de documentos es indispensable tener presentes las normas específicas en gestión documental. Podemos citar la norma UNE-ISO/TR 15489 de gestión de documentos o las ISO 30300 e ISO 30301 (Bustelo, 2011) en fase de aprobación, que permitirán certificar el sistema de gestión de la documentación.

Las normas y su correcta aplicación son una base fundamental para la realización de cualquier proyecto y la creciente importancia de la correcta gestión de la documentación para las organizaciones está comportando la aprobación de sucesivas normas por parte de los organismos responsables.

\section{Nota}

1. En junio de 2011 DLM Forum publicó MoReq2010 http://www.dlmforum.eu

\section{Bibliografía}

1. D’Alòs-Moner, Adela. "Intranets: sonrisas y lágrimas". El profesional de la información, 2002, enero-febrero, v. 11, n. 1, pp. 4-8.

http://www.elprofesionaldelainformacion.com/contenidos/ 2002/enero/1.pdf

2. Cukier, Kenneth. "Data, data everywhere". The economist, 25 Feb. 2010.

http://www.economist.com/node/15557443

3. Bustelo-Ruesta, Carlota. "Norma ISO 30300. Management system for records". Anuario ThinkEPI, 2011, v. 5, pp. 266-268. 